\title{
Effect of Converter Packaging Techniques on Device Electrical Conduction
}

\author{
Angelo L. GATTOZZI, Scott P. PISH, and John A. PAPPAS
}

\begin{abstract}
High power converters handling several thousands of amperes and employing solid state switches (SCRs, for example), often have to use multiple devices in parallel. Additionally, if the converter operates across a high voltage bus, it may also require a series/parallel combination of SCRs. It is clear, therefore, that multiple conducting paths are created in any one leg of the converter. Because of mechanical requirements and practical considerations regarding the mounting of the SCRs and the routing of power to them, differential reactances are generated among the different paths, leading unavoidably to unequal current sharing among the various SCRs. This serious consequence may limit the effective current that can be switched or force the use of larger devices, if this is possible. It is very important, therefore, to be able to estimate the actual current distribution among the various power switches for a given realization of the converter.

This paper discusses the problem as it was experienced in the actual implementation of a three-phase SCR bridge converter. The system geometry is described and the results of the electromagnetic FEA analysis of one leg of the converter are given, highlighting the expected current distribution unbalance. This analysis also points out the extent of skin effects in the various components of the bus-work.

The discussion continues with an analytical and parametric study of the converter leg configuration in order to establish guidelines for the quick estimate of the effect of differential reactances of the various paths and of the excess losses and temperature rise incurred.
\end{abstract}

\section{INTRODUCTION}

A power converter using solid state switches and designed to handle currents in the several thousands of amperes often has to use multiple switching devices in parallel in each of its legs. Furthermore, if the converter operates across a high voltage bus, it may also require a number of switches operating in series in order to handle the voltage stress. Thus, for example, a series/parallel combination of SCRs is often necessary giving rise to a multiplicity of conducting paths in each leg of the converter. The same is true if other types of switches (e.g. IGBTs) are used, although the discussion will be limited in this paper to the implementation with a specific SCR model. Very often mechanical

Manuscript received January 7, 2002. A portion of this research was carried out under the Focused Technology Project sponsored by the U.S. Army Research Laboratory through Science Applications International Corporation under contract 4500002010. Authors are affiliated with The University of Texas at Austin Center for Electromechanics, Austin, Texas 78758, (512) 471-4496, fax (512) 471-0781. requirements and practical considerations regarding the mounting of the SCRs and the routing of power to them preclude the construction of the converter in an electrically balanced way so that differential reactances are generated among the different paths, leading unavoidably to unequal current sharing among the various SCRs. This is a serious consequence that may limit the effective current that can be switched or force the use of larger devices, if this is possible.

This paper presents the results of experience with estimating the actual current distribution among the various SCR switches in a converter designed and built in the laboratory at The University of Texas at Austin Center for Electromechanics (UT-CEM). The SCRs used are model SPT402BHTW360 manufactured by Silicon Power Corporation, and each leg of the converter consists of two branches in parallel, with each branch having three SCRs in series. The converter has the standard configuration of a threephase regenerative ac-dc converter bridge with six legs built as described, and is specified to handle a $10 \mathrm{kV}$ dc bus and a dc bus current of $48 \mathrm{kA}$.

Since all six legs of the converter are identical, it is sufficient to examine only one of them. The interconnections between SCRs and power routings to them are realized with solid aluminum bus bars or plates and solid copper plates are used to interface the aluminum with the anode and cathode surfaces of each SCR. Fig. 1 gives a pictorial representation of the implementation of one converter leg, and Fig. 2 shows the circuital functional diagram.

\section{FEA ANALYSIS}

We have performed an electromagnetic finite element analysis (FEA) under transient conditions of the converter leg shown in Fig. 1 using the Opera-3d commercial software package available from Vector Fields Inc. One simplification was introduced in the model, namely that of treating the sandwich of copper-aluminum-copper ( $\mathrm{Cu}-\mathrm{Al}-\mathrm{Cu})$ plates between two consecutive SCRs in series as a single solid connecting plate with an effective conductivity equal to the weighed conductivities of copper and aluminum based on the length of each material section. Since the primary focus of the research was current distribution in the structure, rather than the switching characteristics of the SCRs, all SCRs were modeled identically as simple conductors with an equivalent conductivity corresponding to their ideal on-state resistance at full-load. Representative results of the FEA analysis are 
shown in Figs. 3 through 6.

Two major results can be derived from the FEA analysis:

- The current distribution in each section is strongly influenced by the skin depth, except for the SCR sections themselves, where (because of the low equivalent conductivity) the current is practically uniformly distributed across the device cross-section. From these results, the effective skin depth is estimated to be $0.25 \mathrm{~cm}$ for the aluminum sections and $0.19 \mathrm{~cm}$ for the copper plates.

- The total current splits unevenly between the two SCR paths in a ratio of roughly $39 \%$ to $61 \%$, so that the bottom series of three SCRs carries a $22 \%$ current overload above the ideal 50-50 split in current. This is expected to translate into almost $50 \%$ higher losses and a corresponding increase in temperature rise.

\section{ANALYTICAL CALCULATIONS}

The equivalent circuit of one converter leg is shown in Fig. 7. Lumped parameters may be used because of the small dimensions of the section of circuit under consideration and the length of the rise time of the exciting waveform. To be noted are the following points:

- $Z_{1}$ represents the impedance of the straight section input and output bus bars shown in Fig. 1 plus the impedance of the feeding lines from the power generator terminals and the internal impedance of the generator itself. Since $Z_{1}$ is much larger than all other impedances shown in the equivalent circuit, the generator can be modeled as an ideal current source with waveform $i(t)$ given by the current waveform measured experimentally through testing. This waveform can be approximated analytically by a trapezoidal wave rising from zero to $i_{0}=43,500 \mathrm{~A}$ in a time of $t_{0}=0.122 \mathrm{~ms}$, remaining constant at the value $i_{0}$ for $0.546 \mathrm{~ms}$ and then decaying linearly back to zero in the same time $t_{0}$.

- $\quad L_{3}$ includes both the inductance of the vertical aluminum post and the additional inductance of the rectangular air space enclosed by the two parallel branches.

With these assumptions, the analytical solution of the circuit equations yields the following results valid up to the time of $t_{1}$ $=0.122+0.546=0.668 \mathrm{~ms}$ :

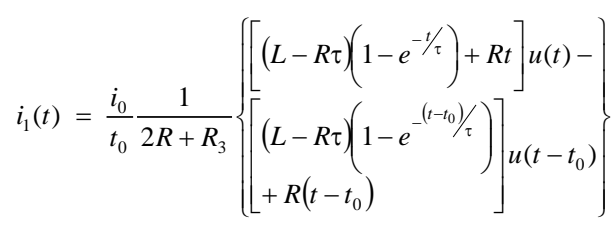

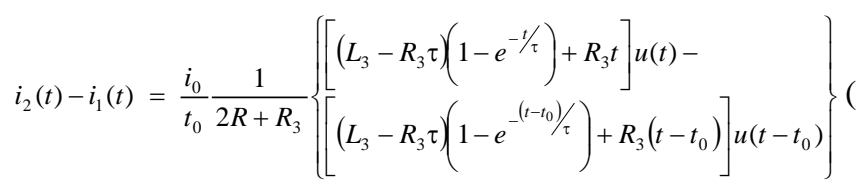

where $u(t)$ is the unit step function and

$$
\begin{aligned}
& R=R_{2}+R_{4} \\
& L=L_{2}+L_{4}+M \\
& \tau=\left(2 L+L_{3}\right) /\left(2 R+R_{3}\right)
\end{aligned}
$$

It can be shown that the current unbalance $i_{2}(t)-i_{1}(t)$ attains its maximum value at either $t_{0}$ or $t_{1}$ depending on the relative values of the circuit components. In order to calculate this current difference, the circuit parameters must first be calculated. The resistances present no difficulty, as long as the effective depth of each section is considered to be equal to the skin depth of the section. The inductances can be estimated with the methods found in references [1] and [2]. Table 1 summarizes the values calculated for our equivalent circuit components.

From these values, it can be calculated that the maximum differential between the two branch currents indeed occurs at $t_{0}$ and that it is equal to $33 \%$ of the maximum current. This is equivalent to predicting a current split of $34 \%$ for $i_{1}$ and $66 \%$ for $i_{2}$.

\section{DISCUSSION}

It is clear from the equivalent circuit as well as intuitive from the physical construction of the converter leg that the major item responsible for the current unbalance is the differential impedance between the two available paths. This is, in fact, also brought out by the analytical solution that shows the strong dependence of the difference between the two currents on the circuit values of $R_{3}$ and $L_{3}$. The role of $L_{3}$ is especially important; Table 2 shows calculated results for some different values of $L_{3}$.

The reduction of $L_{3}$,therefore, should be a primary goal for the minimization of current unbalance. This means that the packaging of the converter leg (routing of conductors, geometry of sections, etc.) must be aimed to strike a suitable compromise between convenience, mechanical requirements, and electrical performance.

\section{CONCLUSIONS}

The leg of a standard topology, ac-dc, three-phase converter bridge, consisting of two parallel branches each having three SCRs in series, was studied to determine the extent of the current distribution unbalance. The results of a FEA simulation show that the current split is in the ratio of $39 \%$ to $61 \%$ between the two branches and that the influence of skin effect in the different conducting sections is pronounced. An analytical formulation of the problem was 
also carried out resulting in the prediction of a $34 \% / 66 \%$ current split. It is reasonable to conclude, therefore, that a current overload of at least $20 \%$ over the ideal $50 \%-50 \%$ current split can be expected for the shorter branch of the leg in the converter. Thus, the SCRs in this leg must be sized to handle higher current and higher di/dt than nominal as well as the concurrent higher losses and temperature rise. The role of the differential reactance between the two legs in determining the magnitude of the overload has also been highlighted and the benefits accruing from of its reduction have been calculated.

\section{ACKNOWLEDGEMENT}

This work was supported by the U.S. Army Research Laboratory and by The University of Texas Institute for Applied Technology.

\section{REFERENCES}

[1] F.W. Grover, Inductance Calculations - Working Formulas and Tables, Dover 1962

[2] S. Ramo, J.R. Whinnery, and T. Van Duzer, Fields and Waves in Communication Electronics, J. Wiley \& Sons, 1967

TABLE 1 Calculated Values

\begin{tabular}{ccc}
\hline \hline Component & Value & Units \\
\hline$R_{2}$ & 12.2 & $\mu \Omega$ \\
$R_{3}$ & 3.2 & $\mu \Omega$ \\
$R_{4}$ & 132.5 & $\mu \Omega$ \\
$L_{2}$ & 0.035 & $\mu \mathrm{H}$ \\
$L_{3}$ & 0.075 & $\mu \mathrm{H}$ \\
$L_{4}$ & 0.0008 & $\mu \mathrm{H}$ \\
$M$ & 0.029 & $\mu \mathrm{H}$ \\
\hline \hline
\end{tabular}

TABLE 2

CALCULATED RESULTS FOR $L_{3}$

\begin{tabular}{ccc}
\hline \hline$L_{3}$ & $i_{1}$ & $i_{2}$ \\
$(\mu \mathrm{H})$ & $(\%)$ & $(\%)$ \\
\hline 0.075 & 34 & 66 \\
0.038 & 40 & 60 \\
0.019 & 44 & 56 \\
\hline \hline
\end{tabular}

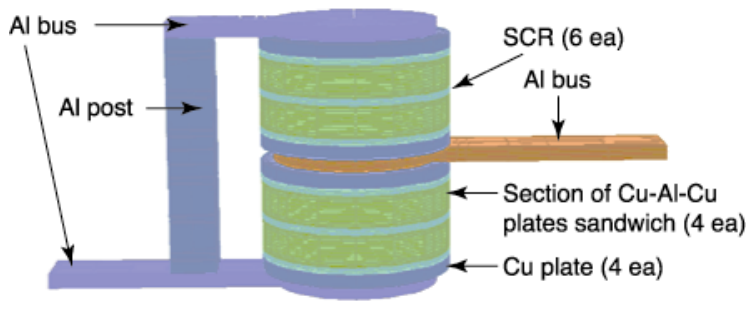

$\mathrm{Al}=$ aluminum, $\mathrm{Cu}=$ copper 3920.0203

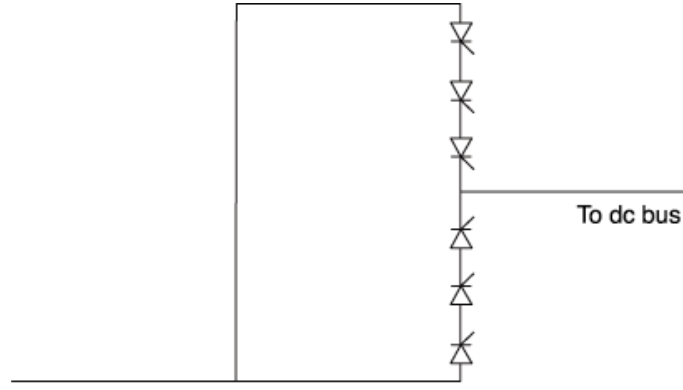

To ac bus

3920.0204

Fig. 2. Functional representation of one converter leg

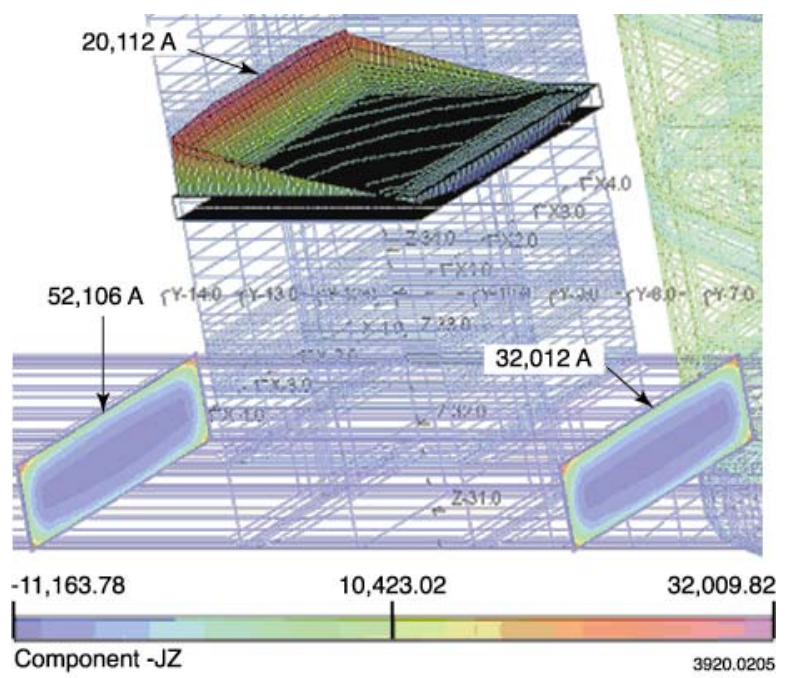

Fig. 3. Current density distribution at two cross-sections of lower bus bar and in vertical post $\left(t=0.1 \mathrm{~ms}, \mathrm{~J}\right.$ units $\left.\mathrm{A} / \mathrm{cm}^{2}\right)$

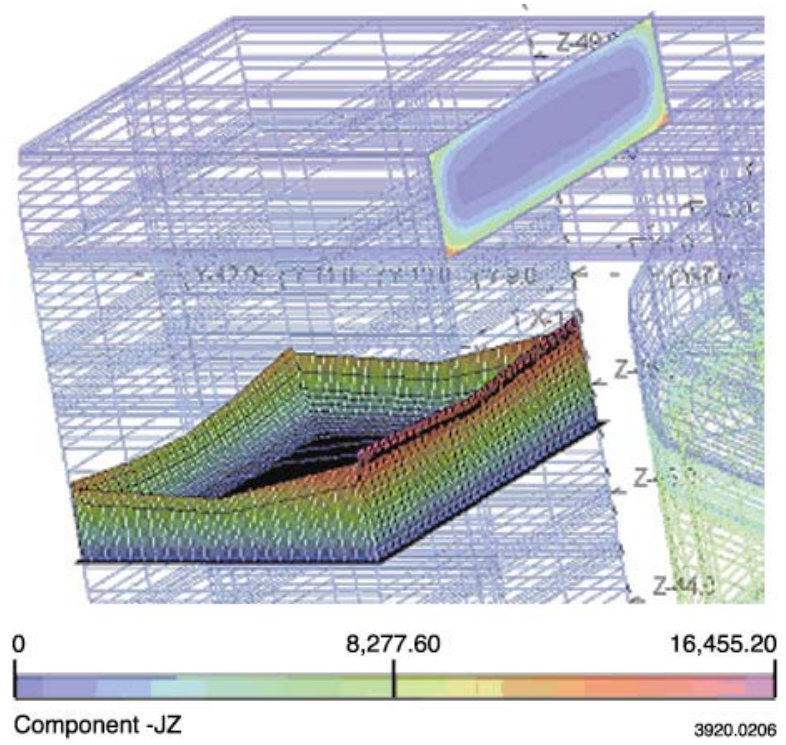

Fig. 4. Current density evolution in vertical post and upper bus bar $\left(t=0.1 \mathrm{~ms}, \mathrm{~J}\right.$ units $\left.\mathrm{A} / \mathrm{cm}^{2}\right)$

Fig. 1. Physical assembly of one converter leg (diameter of an SCR $=12.5 \mathrm{~cm}$ ) 


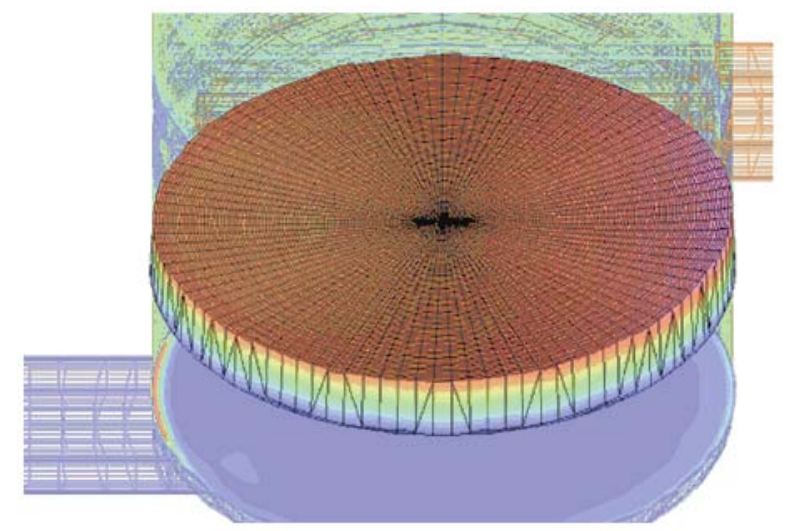

0

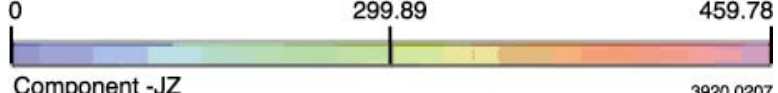

3920.0207

Fig. 5. Current density at midplane across bottom copper plate and at midplane across second SCR from bottom ( $\mathrm{t}=0.1 \mathrm{~ms}$, $\mathrm{J}$ units A/ $\mathrm{cm}^{2}$ )

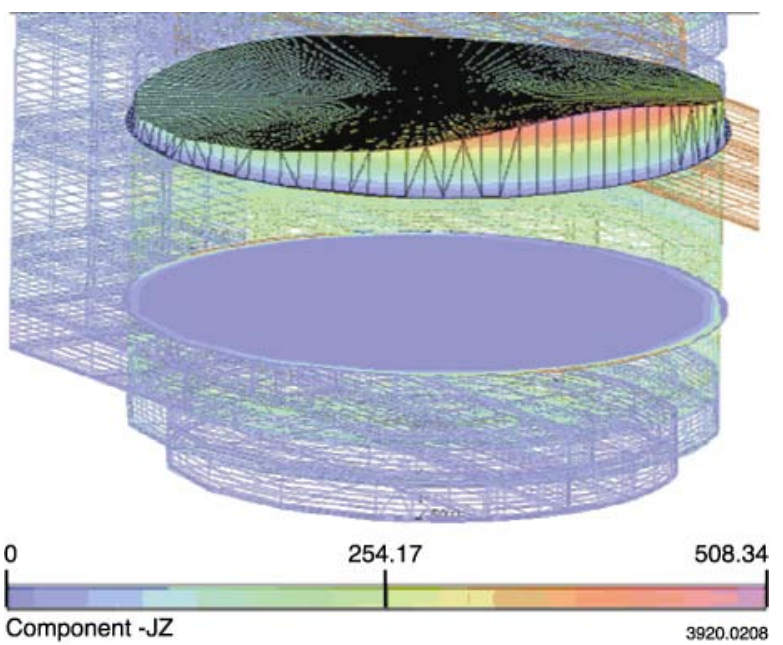

Fig. 6. Current density at midplane across top $\mathrm{Cu}-\mathrm{Al}-\mathrm{Cu}$ sandwich and at midplane across third SCR from top; note that the assembly is shown upside down $\left(\mathrm{t}=0.1 \mathrm{~ms}\right.$, J units $\left.\mathrm{A} / \mathrm{cm}^{2}\right)$

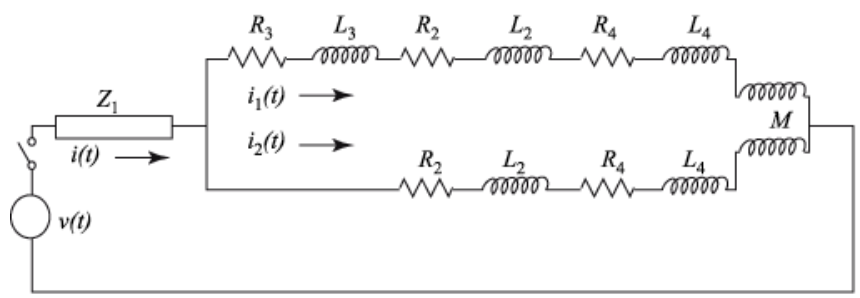

$Z_{1}=$ impedance of generator and input-output bus bars

$R_{2}, L_{2}=$ resistance and inductance of top and bottom A1 bus bars $R_{3}, L_{3}=$ resistance and inductance of vertical post

$R_{4}, L_{4}=$ resistance and inductance of bottom and top switching sections consisting of series combination of two Cu plates, three SCRs, two $\mathrm{Cu}-\mathrm{Al}-\mathrm{Cu}$ sandwich plates

$M=$ mutual inductance of the two parallel branches

3920.0209

Fig. 7. Equivalent circuit of converter leg 